\title{
Lust auf Datenschutz
}

Liebe Leserinnen, liebe Leser,

Glück und Erfolg machen glücklich, aber Glück macht glücklicher. Es macht auch angesehener als Erfolg; der reich Geborene gilt mehr als der Parvenü. Und Unglück ist schlimmer als Misserfolg; eine selbst verschuldete Narbe trägt der Mensch gelassen oder gar mit Stolz; für eine angeborene Entstellung schämt er sich. Glück fällt dem Menschen zu. Ihm nachzuhelfen, mindert es. So geht der Mensch oft ein Risiko ein und setzt auf sein Glück. Das ist nicht vernünftig, denn wenn das Glück ausbleibt, hat er verloren; Vernunft kann das verhindern. Aber Vernunft erfordert Denken; ein durchdachter Entschluss kommt deshalb verzögert an; der Entschluss zum Risiko jedoch ist spontan da.

Das wirkt sich besonders dort aus, wo rasche Entschlüsse gefordert sind, wie z.B. im Umgang mit der Informationstechnik. Sie reagiert unvergleichlich viel schneller als das Gehirn; drängt ihm im Dialog ihren Takt auf; lässt ihm keine Zeit für Vernunft. Also abgesehen davon, dass der Mensch gerne seinem Glück nachgeht, drängt inn die Informationstechnik auch noch zu ungesicherten spontanen Entschlüssen. Kann man es dann dem zum Spiel mit der Technik gelaunten Menschen verdenken, wenn er seine Vernunft ungenutzt lässt, wenn er zum Beispiel auf die Bedingungen von Facebook eingeht und per Einwilligung auf Datenschutz verzichtet? Er gibt dann seine Daten, Vorlieben, Wünsche und Lebensgeschichten preis. Damit macht er sich zum Ziel von Werbung und Ausforschung, von Zumutungen und böswilligen Angriffen aus dem Netz; und aus den vom Netz bedienten Lebensbereichen. Aber trotz der Intensität ist der vernunftraubende Druck der Technik sekundär; denn ohne die Lust des Menschen, der Neugier zu folgen und sein Glück zu versuchen, bliebe der Druck der Technik indifferent und könnte nichts Geordnetes in Gang setzen.

Die Datenschutzaufsichtsbehörden begreifen sich als zuständig; aber ihr Maßnahmenkatalog geht an der Problematik vorbei. Er richtet sich nicht an die Netzteilnehmer sondern an die Betreiber. Diese einerseits und die Aufsichtsbehörden andererseits mögen die gesetzlichen Bestimmungen zunächst unterschiedlich auslegen; aber mit Hilfe eines funktionierenden Rechtsapparats können die Ansichten zur Konvergenz gebracht werden; die Differenzen sind ausgleichbar; sie sind das kleinere Problem. Wenn ein Unbehagen übrig bleibt, dann liegt es daran, dass es in diesem Falle der Geschützte ist, der verantwortlich gegen den Sinn der Schutznorm verstößt; und damit den Sinn des Datenschutzes in Frage stellt. Entweder muss sich also die Norm seinem Verhalten anpassen, oder sein Verhalten muss der Norm angepasst werden. Soll es die letztere Option sein, muss der Teilnehmer auf geeignete Weise dazu gebracht werden, sich gegen die Unbillen des Netzes vorzusehen und sich schützen zu lassen. Die Autoren der DuD-Ausgabe 02/12 sehen darin eine Bildungsaufgabe mit dem Tenor: Man muss dem Teilnehmer das Internet, seine Funktionen, Chancen und auch Gefahren nahe bringen. Wenn er das verstanden und anzuwenden gelernt hat, wird er dankbar auf den Datenschutz zurückgreifen.

Nach den obigen Betrachtungen über Glück und Risiko fragt es sich aber, ob der langlangsame, spröde Verstand ausreicht, die zumeist unbesorgten jugendlichen Teilnehmer dem Spaß entsagen zu lassen. Die Bildungsaufgabe müsste den werbenden Unternehmen wirksam entgegenwirken. Diese sind aber erfahren und wissenschaftlich gerüstet. Sie machen Lust; wogegen es der Bildungsaufgabe zufällt, Angst zu machen. Moral gegen Laster, Vernunft gegen Risiko zu setzen, ist aussichtsarm. Die Bildungsaufgabe hat damit ein Handicap. Doch ein Handicap, liebe Leserinnen und Leser, ist nicht Grund, aufzugeben. Im Gegenteil, es macht klar, dass man sich anstrengen muss. Datenschutz gehört integral, und nicht aufgepfropft, zum Umgang mit sozialen Netzen. Er soll dem Teilnehmer helfen, sich vom Kindesalter an frei und ungefährdet im Netz bewegen zu können. Die Bildungsaufgabe könnte ihm Lust darauf machen, sich geregelt, anerkannt und eventuell vorzeigbar freizuschwimmen.

Mit freundlichen Grüßen, Ihr

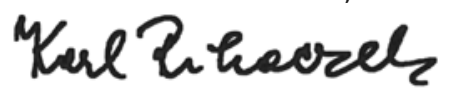

